\title{
How Can Learners Learn from Experience? A Case Study in Blended Learning at Higher Education
}

\author{
$\mathrm{Au}$ Thien Wan
}

\begin{abstract}
E-learning is prevalent and it is changing the learning paradigm in the higher education. This results in less face-to-face contact with educators, lecturers, facilitators and tutors. Therefore capturing and utilizing experiences of learners to make it available or sharable to peers can help to create better environment for learning to improve learning outcomes and quality. Accordingly, learning especially at higher education, involves the interaction of human, technology and knowledge. In this paper, we use the scenario of a typical blended learning at a university setting to highlight the different types of learning experiences that can be harnessed and used for better learning. We also use the scenario to scaffold and formulate a novel framework of blended learning technological architecture to show how to harness learning experiences to be analyzed and possibly be used in a feedback system. We used analytics to produce our output from the analysis of LEs and the visual feedback system received favorable comments from learners.
\end{abstract}

Index Terms-E-learning, learning experience, feedback system, blended learning.

\section{INTRODUCTION}

Traditional face-to-face learning is still the mainstream of education and learning although eLearning has gained a strong foothold in the delivery and the management of courses. With the ubiquity of ICT, proliferation of Internet access and coupled with the many advantages, blended learning is an inevitable trend and a normal course of action in modern educational paradigm. This is because despite the popularity and huge investment in eLearning, the result is still regarded as not quite living up to its expectations and some major concerns in its effectiveness and appropriateness have been revealed in various studies [1], [2]. Many of the eLearning system developed today were merely the automation of the process and management of teaching and delivering of courses with the advantages of eliminating the time and space barrier. The value towards better learning outcomes is still an area of study, although some researchers have recognized the issues and provided innovative solutions to solve some related problems [3], [4].

Blended learning environment is the combination of traditional face-to-face learning and computer mediated or online instruction [5], [6]. In the academic field especially in higher education, the adoption of eLearning is on the rise [7]. As a result of the inevitable paradigm shift in the combination of traditional face-to-face learning and eLearning, also called blended learning, learners'face-to-face contact with

Manuscript received April 14, 2014; revised June 25, 2014.

$\mathrm{Au}$ Thien Wan is with the Computing Information System Department, InstitutTeknologi Brunei, Brunei Darussalam (e-mail: twan.au@itb.edu.bn). educators, lecturers, facilitators and tutors is diminishing. Therefore capturing and utilizing experiences of learners, referred to as learners' experience (LE) throughout the paper, as knowledge available or sharable to peers is crucial anda valuable catalyzein making learning more efficient, producing better outcomes and learning quality. Some researchers have shown improvement of the learning effectiveness in eLearning environment through the sharing of knowledge and experience [8], [9].

Accordingly in a learning environment three components are essential: Human, knowledge and technology (HKT) [10]. This paper defines and highlights the LEs by using a typical higher learning institution environment as a scenario in the context of blended learning setting within the HKT-paradigm. A conceptual framework was formulated to capitalize on the highlighted LE for analysis and for possible use in a graphical feedback system to improve learning. The framework conceptualizes LEs in the form of interactions with the eLearning tools, the lecture and tutorial attendance, and the scores of quizzes at different milestones of the course. LE reuse in the form of knowledge implies the transformation of knowledge to action typically represented as the ability to solve problem and accelerate learning efficiency. Data analyzing techniques were used in order to help optimize peers learning by recommending appropriate LEs to learners based on their behavior. The originality of the concept is the use of data analyzing tools to ultimately recommend LE dynamically to targeted learners based on user profiles and user behavior to optimize the learning process, improve effectiveness and producing better outcomes.

\section{Higher LEARNING INSTITUTION SCENARIO}

In most higher learning institution environment today, a student enrolls at an institute or university in a program, such as a Bachelor of Information Technology, and his or her information is added to the university databases and stored if the student's record does not already exist. In order to obtain a degree from a program, the student is required to complete a number of courses, such as Information Systems, Software Development, Computer Networks, and others. Each course contains a number of study and assessment modules: for example, ER Model, Functional Dependencies, and SQL, to name a few. The student is also presented with a number of learning options as shown in Fig. 1. This is a very common learning scenario in higher learning institutions across the globe.

Students from other programs and faculties may enroll in the same courses, which means they share those courses with students from different departments. For example students from Commerce, Science, Engineering, Arts, Law, and 
others may all enroll in Introduction to Information Systems. As a result, the course has a mix of students from various backgrounds, varying levels of work experience and maturity, and, possibly, different global cultural backgrounds. Obviously, this contributes to student diversity, and consequently to their interests, expectations, and approaches to study.

As a result, each individual learns or interacts differently, despite being presented with the same materials, due to their diverse experiences, maturity levels, and backgrounds. Individual student learning behaviours in terms of attending lectures and tutorials and the milestones they achieve combine to form part of the learning experience (LE) shown in Fig. 1.

The above scenario is therefore capable of providing a platform to engage students in the LE as they proceed through the course. The experience captured includes the:

- Attendances for lecture, tutorials and labs;

- Logs and engagement from the various eLearning elements, for example, eLearning tools, forums logs, and social networking experience; and the

- Milestones achieved.

Using appropriate tools capable of analysing the captured LEs, that is, the interactions of learners with the various learning materials and learning options, interesting learning patterns for each individual learner can be observed. For example, different learning patterns for undergraduates of different programs, different ages, experiences and maturity can be stored and reproduced for students with similar profiles, for whom similar learning patterns would be recommended to achieve better outcomes. Thus, a management student taking the course could be recommended to follow a similar learning pattern to another successful student from the management program or a Science major could be recommended to follow the successful learning patterns of a similar student from the same program.

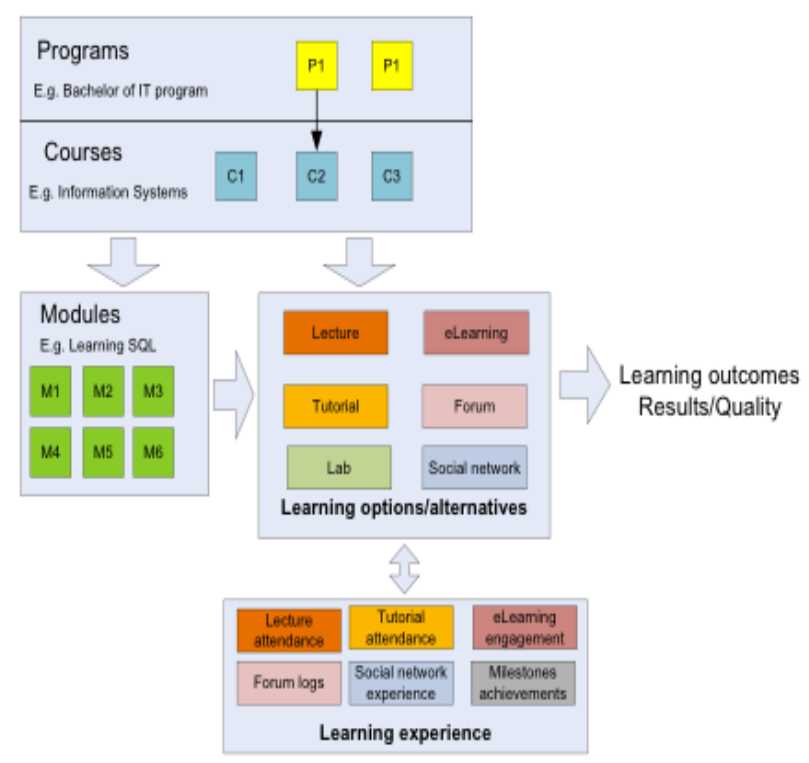

Fig. 1. A blended learning scenario in a university.

\section{TECHNOLOGICAL FRAMEWORK OF LES}

The scenario above forms the background of the technological framework through which the analyses and recommendations can be made. Fig. 2 shows the Learning Experience Technology Framework and the core components of the system are the eLearning tool, back-end database system, data analysis tools, and feedback and recommender system. The framework also depicts a blended learning environment in which traditional learning and eLearning taking place simultaneously. The key factors underlying this framework are the capture of LEs from various sources within the context of the blended learning environment and, with the help of data analysis tools, the recommendation of LEs to peers with similar profiles. These profiles might include features such as the learners' learning metrics, including attendance or performance outcomes at specific milestones, or instances of engagement with various eLearning components, for instance, interactions at specific time intervals or the frequency of interactions. Using appropriate tools capable of analysing the captured LEs, that is, the interactions of learners with the various learning materials and learning options, interesting learning patterns for each individual learner can be observed.

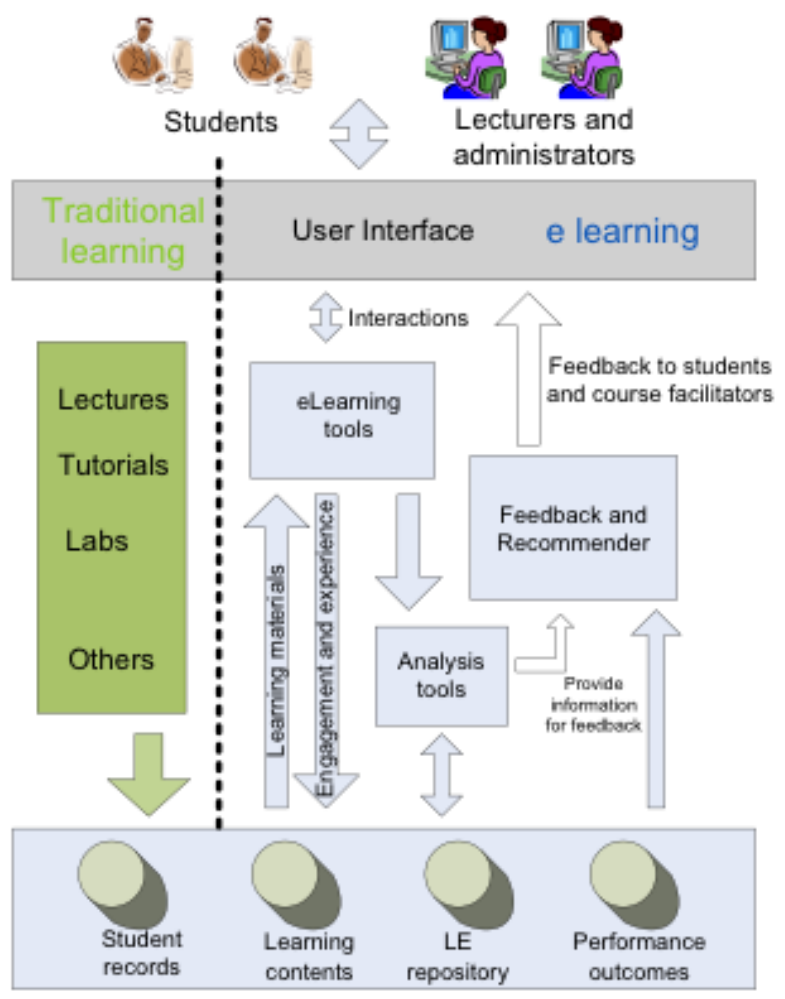

Fig. 2. Blended learning experience technology framework.

\section{A. The Databases}

The database consists of the learning content, LE repository, student record, and the performance/outcomes.

The learning contents are learning objects uploaded by facilitators or module coordinators in authority. Users, both module coordinators and learners, interact with learning objects through the eLearning system.

LE Repository stores all the raw captured engagement data $(\log s)$, as well as the analysed LEs from the analysis tools. The logs are critical in the sense that they represent one of the main experiences the students have in the learning process. The logs are the captured LEs from the students' engagement with the eLearning tool. The data structures of the logs are able to link to student records, students' output performance data and other information for analysis and, ultimately, 
providing feedback and recommendations. Essentially, the $\log$ also consists of students' ID, semesters of study, topics studied, questions answered, and the time stamp. The ID allows the data structure to link to other student information and records. The topics and questions are recorded in the learning instances. The time stamps indicate the time when the engagement of students with the eLearning tool takes place. When the analysis is initiated, all these pieces of information synthesize together to form the learning patterns, interactive experiences and cognitive behaviours of students.

Student records store personal information about the learner, such as name, gender, age, student type, department, learning goals or objectives, and so on. This is usually created when a learner enrolls in a course.

Performance and outcomes store the results of a learner's learning competency in tests, quizzes, exams, and possibly past awards the learner has received.

\section{B. Analysis Tools}

The analysis involves several tools to generate the required outputs. The students' engagement logs are linked to the resources residing in the backend databases, such as student records and student performance data, in order for the analysis to generate the desired output.

A relational database (MySQL) is used to link and organize all the logs and related data resources to facilitate management and manipulation of the data by the analysis tools. The main tools used are SPSS and PLS statistical packages, which are able to generate crucial statistical outputs. These tools also help in the classification of data, the discovery of associations and correlation between data, the characterization and summarization of data, the discovery of discriminant features, and the identification of outliers, to name a few.

Algorithms were developed to do data integration, data selection, and finally, the evaluation of discovered patterns, using simple visualization. Interesting and potentially useful patterns are also extracted from the integrated data sets to discover useful learning patterns in the form of LEs with deduced hypotheses that can be reused by other learners to improve their learning outcomes.

Fig. 3 shows the process of databases from different sources being fed to respective analysis tools to generate various forms of output before being used for feedback and recommendation.

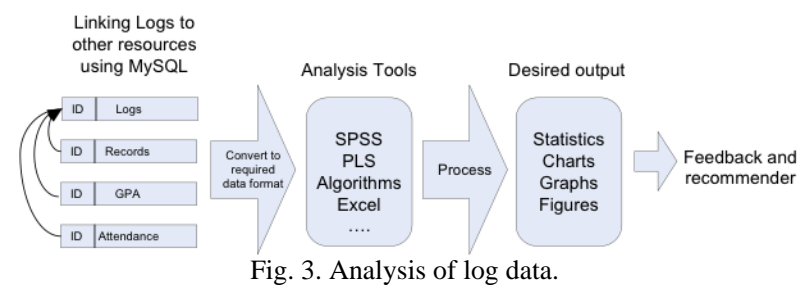

\section{Feedback and Recommender}

Studies have shown that providing feedback and recommendations can increase user confidence and have positive influence on the attitude of users towards a system [11], [12]. Unlike recommenders in e-commerce that have been around longer due to their direct, immediate and lucrative benefits to businesses, recommenders in eLearning have only started to take shape as recently as the early millennium. A recommender system in an eLearning system usually recommends a learning task to a learner based on tasks already done by the learner and their successes, and based on tasks made by other 'similar' learners. The similarity of learners is established using user profiles, or may be based on common previous access patterns. A recommender system suggests possible actions or web resources based on its understanding of the user's access. Fig. 4 shows the process of the feedback system.

The approach we suggest in our framework is to use a relational database that links up and takes into account the records and performances of learners, their access history in the forms of LEs, and simple SQL commands can be used together with the pre-set recommendation criteria for the recommender systems. With output from the analysis tools, these techniques can identify learners and find an 'association' with users to best fit the recommended criteria and produce timely feedback for learners. The feedback can be in many forms and we include visual feedback to provide a more direct visual impact for the learners.

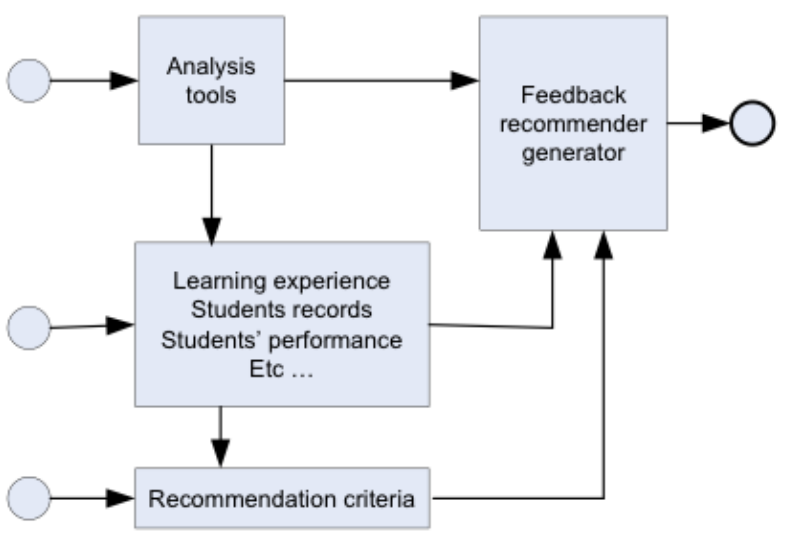

Fig. 4. Flow of feedback and recommender system.

\section{Visual Feed Back}

Kiviat diagram has common application in the control of quality improvement to display the performance metrics of any ongoing program [13]. It is a graphical method of displaying multivariatedata in the form of a two-dimensional diagram of three or more quantitative variables represented on axes starting from the same point.

A Kiviat diagram consists of a sequence of angular spokes, called radii, with each spoke representing one of the variables. The data length of a spoke is proportional to the magnitude of the variable for the data point relative to the maximum magnitude of the variable across all data points. The plot is star-like in appearance when the data points are connected. The relative position and angle of the axes is typically uninformative. Fig. 5 and Fig. 6 show examples of Kiviat diagram of used in our system.

Kiviat diagrams are a useful way to display multivariate observations with an arbitrary number of variables [14]. Each graph represents an instance of a static observation. The beauty of the Kiviat diagram is that they are easy to read, even for untrained personnel; it is easier to see patterns in the data, which are visually striking, and can add interest to what would otherwise be a dry data presentation. Hence, the Kiviat diagram is selected to provide visual feedback and examine the impact of feedback instruments on changes in study patterns in general and eLearning interactions specifically. 


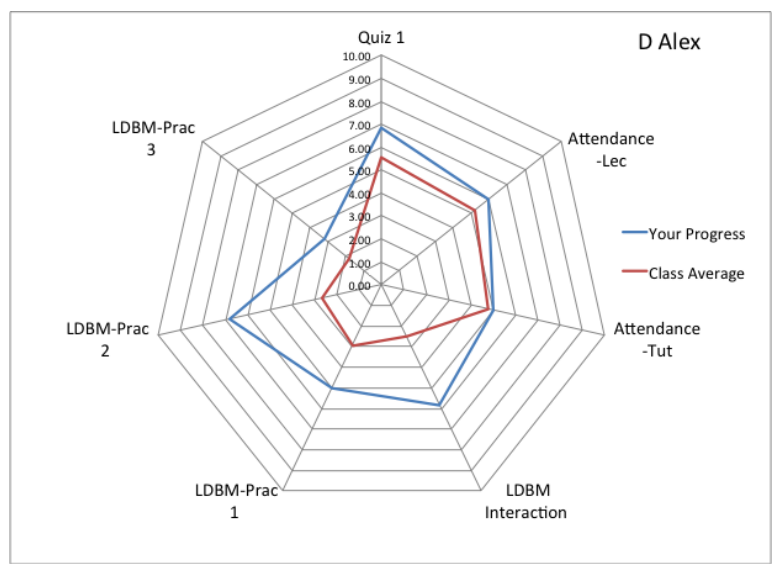

Fig. 5. Kiviatdiagram showing D Alex's metrics.

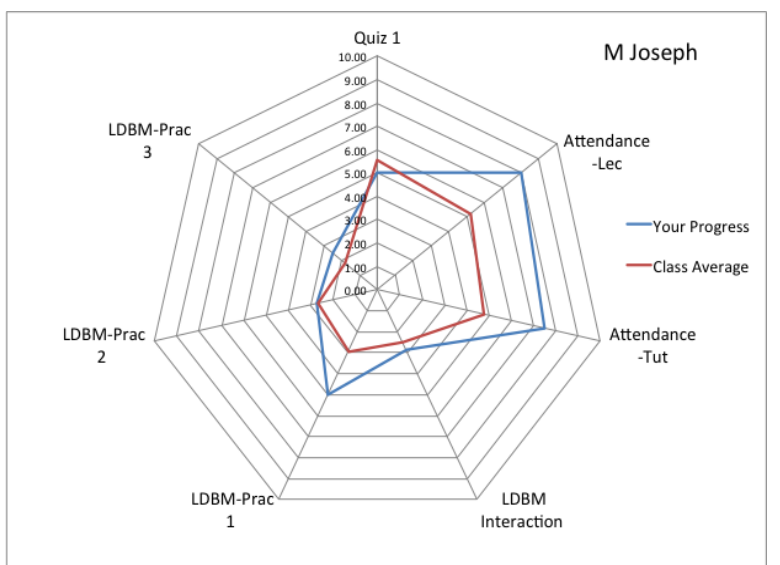

Fig. 6. Kiviatdiagram showing M Joseph's metrics.

In Fig. 5, student D Alex's (not his real name) learning metrics were all above class average. For example, his Quiz 1 score is 7 and his engagement with the LDBM Interactionof eLearning components is higher than average. On the other hand, in Fig. 6, student M Joseph's (not his real name) performance was somewhat unsatisfactory. We recommend that he should engage with the eLearning component (LDBM Interaction) more often throughout the course, especially towards the milestones. We also used Kiviat diagrams to highlight what components of the course s/he needed to catch up with, for instance, lecture attendance and engagement with LDBM-Prac3. Further, we recommend the student to pay more attention to those particular components or we would direct them to the right learning paths: for instance, the recommendation for D Alex:

"You are doing well. You could improve further by trying the online LDBM more, in particular, for concept 3. Attending lectures and tutorials more regularly should also help you understand better."

As for the teaching staff, the results analysis provided some valuable feedback. For instance, the class average for all metrics (blue color line) in the Kiviat diagram provides some insightful information on the effectiveness of the tool for possible pedagogy innovation and improvement too. The class average shown in the Kiviat diagram could allows the learner to make appropriate adjustments to his learning behaviour.

The comments from the students about the Kiviat diagrams are very positive. Here are some of the excerpts:

"The system is useful and it's the first subject where I've seen this information actually graphed."

"I thought this was a great feature of the course. It'd be great to see this across other subjects as it's great to see how you stack up against the rest of the class. Great work there; I thoroughly admired it."

"It's very useful. I think all courses should have this. It's very well done."

By providing recommendations together with the Kiviat diagram our system could:

- Motivate the learner when working in the course so s/he does not get frustrated if the results are lower than expected;

- Enable collaboration: fosters sharing of contributions, communicating with course members, etcetera; and

- Promote self-reflection through visualisation of the learning metrics.

\section{CONCLUSION}

The paradigm shift in education in higher learning institution is inevitable and challenging. It also presents many opportunities for educators, learners, facilitators and management staffs to work closely and improve learning outcomes and quality. In a typical university scenario and with the proposed Learning Experience Technology Framework, many types of LEs can be harnessed and used in a meaningful and productive way to produce better learning outcomes and quality. It is apparent that in our proposed framework it can easily be adapted to similar setting in any higher learning institutions. The recommended feedback from the students also reflects favourable inclination towards learning after using the system in terms of innovations, comparisons amongst peers learners, and usefulness as a whole.

The proposed prototype is not without limitations and it can be improved in many ways. For instance we could include a more complex dynamism in the interaction of learners though eLearning tools using other popular social networks and harnessing the LEs to study the social interaction patterns contributing to learning. Other sociological and psychological factors can also be looked into to investigate the engagement of learners with the system. In the proposed framework we use very simple if-then statement based on eLearning log data, grades, and attendances to make selection on a predefined criteria. We could in the future include a more complex recommendation system using advanced data mining techniques. Although learners' profile, learning patterns and background were collected, they were not fully used in our system yet. This could alsoprovide a very rich ground for future research topics. And we see a great potential in further developing the system into a full-blown experience learning feedback system.

\section{REFERENCES}

[1] P. R. Polsani, "Use and abuse of reusable learning objects," Journal of Digital Information, vol. 3, no. 4, pp. 2002-2003, 2003.

[2] C. B. Teo and R. K. L. Gay, "A knowledge-driven model to personalize e-learning," ACM Journal of Education Resources in Computing, vol. 6, no. 1, Article 3, 2006.

[3] P. Brusilovsky, Knowledge Tree: A Distributed Architecture for Adaptive e-Learning, New York, USA, 2004. 
[4] P. Dolog, B. Simon, W. Nejdl, and T. Klobucar, "Personalizing access to learning networks," ACM Transactions on Internet Technology, vol 8 , no. 2, p. 3, 2008.

[5] D. R. Garrison and N. D. Vaughan, Blended Learning in Higher Education: Framework, Principles, and Guidelines, John Wiley \& Sons, 2008.

[6] C. J. Bonk and C. R. Graham, The Handbook of Blended Learning: Global Perspectives, Local Designs, John Wiley \& Sons, 2012.

[7] A Gunasekaran, R. D. McNeil, and D. Shaul, "E-learning: research and applications," Industrial and Commercial Training, vol. 34, no. 2, pp. 44-53, 2002.

[8] D. Helic, H. Maurer, and N. Scerbakov, "Knowledge transfer processes in a modern WBT system," Journal of Network and Computer Applications, vol. 27, no. 3, pp. 163-190, 2004.

[9] D. Helic, "Formal representations of learning scenarios: A methodology to configure e-learning systems," Journal of Universal Computer Science, vol. 13, no. 4, pp. 504-531, 2007.

[10] A. T. Wan, S. Sadiq, and X. Li, "Exploratory study on learners' experience in an eLearning system," in Proc. $17^{\text {th }}$ American Conference on Information Ssystems(AMCIS 2011), Detroit, USA, August 2011.

[11] K. Swearingen and R. Sinha, Beyond Algorithms: An HCI Perspective on Recommender Systems, 2001, Citeseer.
[12] O. C. Santos and J. G. Boticario, "Improving learners' satisfaction in specification-based scenarios with dynamic inclusive support," in Proc. 8th IEEE International Conference on Advanced Learning Technologies (ICALT'08), 2008, Santander, Cantabria, Spain.

[13] R Basu, Implementing Quality: A Practical Guide to Tools and Techniques: Enabling the Power of Operational Excellence, Thomson London, 2004

[14] J. M. Chambers, Graphical Methods for Data Analysis, Wadsworth, 1983

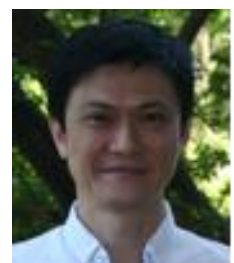

Au Thien Wan is a senior lecturer with the Department of Computing and Information System, the Faculty of Business and Computing (FBC), Institut Teknologi Brunei, Brunei Darussalam. He is also the deputy dean of FBC. He holds BEng (EEE) from Glasgow University, UK, MSc in data communication system from Brunel University, UK and $\mathrm{PhD}$ from Queensland University, Australia.

He has also has published articles in AMCIS, PACIS and ACIS in the area of e-learning in particularin learning experience. 